ISSN 0103-5150

Fisioter. Mov., Curitiba, v. 24, n. 1, p. 87-97, jan./mar. 2011 Licenciado sob uma Licença Creative Commons

\title{
Análise cinemática e eletromiográfica do alcance em pacientes com acidente vascular encefálico
}

\author{
Kinematic and electromyographic analysis of the reaching in \\ stroke patients
}

\author{
Karoline Cipriano Raimundo ${ }^{[\mathrm{a}]}$, Laís Sampaio Silveiraa ${ }^{[\mathrm{a}]}$, Marcos Seizo Kishi ${ }^{[\mathrm{b}]}$, \\ Luciane Fernanda Rodrigues Martinho Fernandes ${ }^{[c]}$, Luciane Aparecida Pascussi Sande Souza ${ }^{[d]}$ \\ [a] Acadêmicas de Fisioterapia da Universidade Federal do Triângulo Mineiro (UFTM), Uberaba, MG - Brasil, \\ karolelais@gmail.com \\ [b] Docente do curso de Fisioterapia da Universidade Estadual Paulista Júlio de Mesquita Filho, Doutor e Mestre em \\ Fisioterapia pela UFSCar, linha de pesquisa: Avaliação de agentes histaminérgicos sobre a ansiedade, aprendizagem e \\ memória de camundongos, Uberaba, MG - Brasil. \\ [c] Docente do curso de Fisioterapia da Universidade Federal do Triângulo Mineiro, Mestre em Bioengenharia pela USP, \\ Doutora em Educação Física pela Universidade Estadual de Campinas (Unicamp), Uberaba, MG - Brasil. \\ [d] Docente do curso de Fisioterapia da Universidade Federal do Triângulo Mineiro, Mestre em Fisioterapia pela UFSCar, \\ Doutora em Controle Motor pela Universidade Estadual de Campinas (Unicamp), Uberaba, MG - Brasil.
}

\section{Resumo}

Introdução: O movimento de alcance é muito estudado na literatura, no entanto, poucos estudos realizam análise cinemática e eletromiográfica desse movimento em sujeitos hemiparéticos. Objetivo: Avaliar o alcance de indivíduos hemiparéticos pós-acidente vascular encefálico (AVE) em seus aspectos cinemáticos e eletromiográficos. Materiais e métodos: Foram selecionados quatro indivíduos, idade média de 54,5 \pm 10,7 anos, com diagnóstico de AVE associado à hemiparesia. Realizou-se a avaliação cinemática e eletromiográfica concomitantemente do movimento de alcance na postura sentada, tanto do lado parético quanto do não parético. Resultados: Houve diferença significativa entre os sujeitos com relação ao deslocamento $\left(F_{(3,63)}=3.081, p=0.03\right)$, porém, não ocorreu diferença significativa entre os lados $\left(F_{(1,63)}=\right.$ $1.441, \mathrm{p}=0.23$ ). Com relação às articulações (ombro, cotovelo e punho), houve diferença significativa entre os deslocamentos de cada uma $(F(2,63)=27.496, \mathrm{p}=0.00)$, assim como entre as coordenadas $\mathrm{x}, \mathrm{y}$ e $\mathrm{z}$ $\left(F_{(2,63)}=36.702, p=0.00\right)$. Na análise dos dados eletromiográficos, não houve diferença significativa entre 
os sujeitos $\left(\mathrm{F}_{(3,31)}=2.437, \mathrm{p}=0.08\right)$, entre os lados $\left(\mathrm{F}_{(1,31)}=3.384, \mathrm{p}=0.07\right)$ e entre os músculos $\left(\mathrm{F}_{(4,31)}=\right.$ $0.942, \mathrm{p}=0.45$ ). Existiu diferença no tempo de execução dos movimentos quando comparado o lado não acometido com o acometido. Conclusão: As análises cinemática e eletromiográfica, de um modo geral, mostraram resultados semelhantes aos encontrados na literatura. No entanto, este estudo acrescenta uma visão mais sistematizada do movimento de alcance, considerando seus aspectos funcionais, diferindo de outros estudos por apresentar, concomitantemente, análises cinemática e eletromiográfica e por investigar ambos os membros superiores.

Palavras-chave: Acidente vascular encefálico. Hemiparesia. Cinemática. Eletromiografia.

\section{Abstract}

Objective: The reaching movement has been extensively studied in the literature. However, few studies have performed kinematic and electromyography analysis of this movement in hemiparetic subjects. Objective: To evaluate the reaching in hemiparetic subjects post-stroke in their kinematic and electromyographic aspects.

Materials and methods: Four individuals were selected, with mean age of $54.5 \pm 10.7$ years old, diagnosed with stroke associated with hemiparesis. We conducted an assessment of kinematic and electromyography concurrently during the reaching movement in a sitting posture. Both paretic and non-paretic sides were tested. Results: Statistical differences were found between subjects with respect to displacement $\left(F_{(3,63)}=3.081\right.$, $p=0.03)$ but no significant difference between the sides investigated $\left(F_{(1,63)}=1.441, p=0.23\right)$. With respect to joints (shoulder, elbow and wrist), there was a significant difference in the displacement of each one $\left(F_{(2,63)}=\right.$ $27.496, p=0.00)$ as well as between coordinates $x, y$ and $z\left(F_{(2,63)}=36.702, p=0.00\right)$. In the electromyographic analysis, no significant difference were found between subjects $\left(F_{(3,31)}=2.437, p=0.08\right)$, between sides $\left(F_{(1,31)}=\right.$ $3.384, p=0.07)$ and between muscles $\left(F_{(4,31)}=0.942, p=0.45\right)$. There was a difference in the time of movement execution when comparing the paretic with non-paretic side. Conclusion: The kinematic and electromyographic analysis, in general, showed results similar to those found in the literature. However, this study adds a systematic vision of the reaching movement, considering functional aspects, differing from other studies to present, simultaneously, kinematic and electromyographic analysis and investigate both upper limbs.

Keywords: Stroke. Hemiparesis. Kinematic. Electromyography.

\section{Introdução}

As doenças encefalovasculares constituem a terceira causa de óbito e a primeira causa de incapacidade física no mundo, dentre elas destaca-se o acidente vascular encefálico (AVE). (1). Após a ocorrência do AVE, geralmente há presença de incapacidades residuais musculoesqueléticas, alterações sensoriais e cognitivas, com impacto potencial na utilização do membro superior e na execução de atividades básicas da vida diária (alimentar-se, vestirse, pentear-se) e de atividades instrumentais (gerenciamento doméstico, mobilidade na comunidade) (2). Durante as atividades que envolvem o membro superior, mesmo em indivíduos com quadro leve de hemiparesia, os movimentos de alcance no membro acometido são lentificados e limitados pela amplitude de movimento, com a trajetória segmentada e a coordenação entre articulações interrompida, mesmo comparando-se aos indivíduos saudáveis (3).

Esse alcance tem sido bastante explorado na literatura (4-11). Há mais de duas décadas, Jeannerod $(12,13)$ propôs a mais detalhada descrição do movimento de alcance em sujeitos saudáveis. Segundo ele, a mão segue uma trajetória característica enquanto se move em direção ao objeto. Essa fase é conhecida como transporte.

Em comparação com as escalas de avaliação clínica, estudos de cinemática oferecem uma avaliação sensível e quantitativa dos componentes do desempenho motor anormal (14). Durante a última década, estudos cinemáticos em pessoas com hemiparesia crônica produziram informações importantes sobre como o controle dos movimentos são alterados após o acidente vascular encefálico e também sobre estratégias compensatórias do controle dos 
movimentos (15). Alguns estudos comparam o comportamento da função motora da mão em sujeitos saudáveis (9-11), outros avaliam as estratégias compensatórias $(6,14-17)$, medem mudanças no desempenho ao longo do tempo (14), se concentram na coordenação temporal entre os componentes do movimento (18) e outros, ainda, avaliam as mudanças induzidas pela terapia (19-24).

Além dos dados cinemáticos, a análise eletromiográfica se faz necessária para uma avaliação mais detalhada da função muscular. No entanto, poucos estudos avaliaram a atividade motora de indivíduos com hemiparesia utilizando essa ferramenta. Estudos voltados para membros inferiores (25-27) superam em número aqueles sobre a extremidade superior e, nesse último caso, existem estudos realizados em indivíduos saudáveis (28), enquanto em indivíduos hemiparéticos os estudos não integram todas as articulações do membro superior $(29,30)$.

Embora vários autores tenham investigado o movimento de alcance nos hemiparéticos, ainda existem algumas lacunas que precisam ser preenchidas:

1) comparar os movimentos do membro acometido com o não acometido;

2) investigar os movimentos das várias articulações envolvidas nessa atividade (ombro, cotovelo, punho);

3) associar medidas de atividades musculares, explorando os principais músculos ativos durante $o$ alcance.
Portanto, o objetivo deste estudo foi avaliar o alcance de indivíduos hemiparéticos em fase inicial pós-AVE em seus aspectos cinemáticos (deslocamento e tempo total) e eletromiográficos (RMS).

\section{Materiais e métodos}

\section{Sujeitos}

Participaram desta pesquisa quatro indivíduos (três do sexo feminino e um do sexo masculino), idade média de 54,5 \pm 10,7 anos, com diagnóstico de AVE (três isquêmicos e um hemorrágico) associado à hemiparesia (Tabela 1). Todos foram encaminhados pelo Setor de Neurologia do Ambulatório Maria da Glória da Universidade Federal do Triângulo Mineiro (UFTM) e pelo Centro de Reabilitação Professor Dr. Fausto da Cunha Oliveira. Este projeto foi aprovado pelo Comitê de Ética em Pesquisa da UFTM (Protocolo n. 1490) e os pacientes que concordaram em participar da pesquisa assinaram o Termo de Consentimento Livre e Esclarecido. Todos estavam sob acompanhamento médico, com tratamento medicamentoso individualizado, e foram incluídos na amostra somente aqueles que preencheram os critérios de inclusão.

Os critérios de inclusão foram: idade entre 40 e 70 anos; diagnóstico de AVE com quadro de hemiparesia há mais de três meses e há menos de um ano (com deficiência da função motora do membro superior,

Tabela 1 - Caracterização dos sujeitos da pesquisa

\begin{tabular}{lccccc}
\hline Sujeito & $\mathbf{1}$ & $\mathbf{2}$ & $\mathbf{3}$ & $\mathbf{4}$ & Média \pm desvio-padrão \\
\hline Idade & 46 & 56 & 47 & 69 & $54,5 \pm 10,7$ \\
Sexo & $\mathrm{F}$ & $\mathrm{F}$ & $\mathrm{F}$ & $\mathrm{M}$ & $\mathbf{-}$ \\
Dominância & $\mathrm{D}$ & $\mathrm{E}$ & $\mathrm{D}$ & $\mathrm{D}$ & - \\
Tipo AVE & $\mathrm{I}$ & $\mathrm{H}$ & $\mathrm{I}$ & $\mathrm{I}$ & - \\
Local AVE & $\mathrm{CID}$ & $\mathrm{NIP}$ & $\mathrm{CIE}$ & Frontal lacunar D & - \\
Tempo pós-AVE & 6 meses & 10 meses & 6 meses & 6 meses & $7 \pm 1,7$ \\
Escore Fugl Meyer & 223 & 215 & 216 & 211 & $215,25 \pm 3,6$ \\
Escore EDT & 16 & 20 & 23 & 16 & $18,75 \pm 2,9$ \\
Escala Ashworth & 1 & 1 & 1 & 0 & $0,75 \pm 0,4$ \\
\hline
\end{tabular}

Legenda: $\mathrm{F}=$ feminino; $\mathrm{M}$ = masculino; $\mathrm{D}=$ direita; $\mathrm{E}=$ esquerda; $\mathrm{I}=$ isquêmico; $\mathrm{H}$ = hemorrágico; $\mathrm{CID}=$ cápsula interna direita; $\mathrm{CIE}=$ cápsula interna esquerda; NIP = não informado no prontuário. 
mas capaz de realizar movimentos de alcance); escore da Fugl-Meyer > que 50 (31); com nível cognitivo adequado para entender os procedimentos e as orientações dadas; e ter assinado, após a leitura do termo de esclarecimento, o termo de consentimento livre e esclarecido para a participação no estudo.

Foram excluídos do estudo indivíduos com hemiplegia, escore da Fugl-Meyer $<50$, comprometimento cognitivo importante e com patologias do sistema musculoesquelético e neurológico, além de queixas incapacitantes nesses sistemas, e aqueles que não compareceram na avaliação.

\section{Materiais}

Para a coleta dos dados foi utilizado um eletromiógrafo da marca EMG System do Brasil com seis canais; duas filmadoras DVD SC-DX 103 da marca Samsung com obturador manual; dois tripés; máquina fotográfica com flash para sincronização das coletas; calibrador para filmagem; e computador com 4 GB. Para os posicionamentos foram utilizados: goniômetro, fita métrica, lápis dermográfico, eletrodos para a EMG e pérolas.

Para todos os sujeitos foi preenchida uma ficha de avaliação fisioterapêutica contendo: informações referentes aos dados pessoais; anamnese; dados vitais; exame físico; diagnóstico fisioterapêutico; escala Fugl-Meyer em português para avaliar controle motor dos membros, sensibilidade superficial e profunda e equilíbrio de cada indivíduo; escala de deficiência de tronco (EDT) para avaliar a capacidade de permanecer na postura sentada, já que esta era exigida para a posição inicial do movimento analisado; escala de Ashworth modificada para avaliação do tônus.

\section{Procedimento}

Inicialmente foi preparada a sala de coleta, de modo a deixar os marcadores "pérolas" mais visíveis e evitar ruídos que interferissem no sinal eletromiográfico. Esses marcadores foram confeccionados com pérolas de bijuteria de $0,70 \mathrm{~cm}$ de diâmetro. As paredes, a cadeira e o local do alvo foram revestidos com tecidos pretos e as luzes foram apagadas. Foi construído um calibrador de ferro de $130 \times 100 \times 70$ (altura, largura e profundidade), também pintado de preto, onde foram colocados os marcadores "pérolas" em distâncias de 10 em $10 \mathrm{~cm}$. Por meio dele foi criado um arquivo de referência com as coordenadas $\mathrm{x}$, y e $\mathrm{z}$ a partir da sua filmagem pelas duas filmadoras.

A posição inicial da coleta foi com o voluntário sentado a $90^{\circ}$ de flexão de quadril, joelho e tornozelo; tronco estabilizado no encosto da cadeira usando uma faixa na altura do peito para minimizar movimentos compensatórios; ombro em neutro e cotovelo em flexão de $75^{\circ}$ a $90^{\circ}$. Em dois casos, foi utilizado um apoio individualizado nos pés para obter o posicionamento adequado. 0 alvo era um marcador tipo pérola sobre um tecido preto que recobria uma estrutura posicionada na altura do processo xifoide de cada voluntário. A distância foi o comprimento funcional do braço (da linha axilar anterior até a prega do punho quando o indivíduo eleva seu braço mais próximo a $90^{\circ}$ de flexão possível (24) no plano sagital) (32), conforme pode ser visualizado na Figura 1.

As duas câmeras de vídeo foram apoiadas nos tripés com angulação de $50^{\circ}$ entre elas e, para a reconstrução tridimensional, cada marcador deveria ser visível por ambas simultaneamente. A filmagem foi realizada a uma frequência de $60 \mathrm{~Hz}$. Os marcadores tipo pérola foram posicionados no ombro (tubérculo maior do úmero), no cotovelo (epicôndilo lateral) e no punho (processo estiloide do rádio).

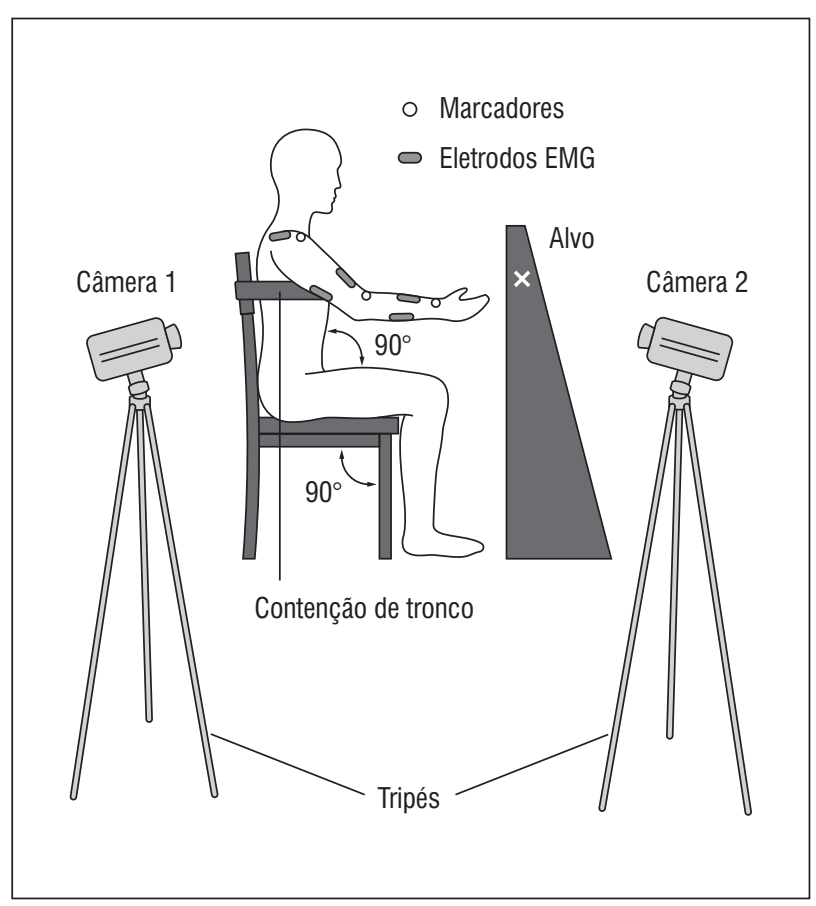

Figura 1 - Esquematização do ambiente de coleta 
Ao mesmo tempo foi registrada a atividade eletromiográfica dos músculos trapézio superior, bíceps, tríceps, flexor ulnar do carpo (FUC) e extensor radial do carpo (ERC) pelo eletromiógrafo (EMG System do Brasil Ltda) de seis canais para EMG. Suas especificações são: passa-banda de 20 a 500 $\mathrm{Hz}$, com rejeição de modo comum $>120 \mathrm{~dB}$, impedância de entrada > 10 Mohms e ganho de 100x no condicionador de sinais e de $20 x$ no eletrodo bipolar passivo, totalizando $2000 \mathrm{x}$.

A colocação e o posicionamento dos eletrodos seguiram as determinações do protocolo Surface Electromyography for the Non-invasive Assessment of Muscles (Seniam) (33), que preconiza que, para a diminuição da impedância, deve ser feita a tricotomia do local, com posterior desengorduramento com álcool e retirada da camada de células mortas com uma lixa fina seguida de limpeza com álcool. Somente após esses procedimentos os eletrodos foram fixados na pele e durante a coleta todos os aparelhos eletrônicos locais e em salas vizinhas foram desligados.

0 eletrodo, no trapézio superior, foi colocado a $50 \%$ da linha do acrômio e do processo espinhoso da vértebra $\mathrm{C} 7$. No bíceps, na linha medial entre o acrômio e a fossa cubital, a 1/3 desta. No tríceps, foi colocado sobre a linha entre a crista posterior do acrômio e o olécrano (33). Para o posicionamento dos eletrodos no FUC e no ERC foram marcados dois eixos no antebraço, sendo um medial denominado de eixo longitudinal medial (ELM) para o flexor e um lateral denominado de eixo longitudinal lateral (ELL) para o extensor. Os dois eixos horizontais se estendiam, com o antebraço em posição neutra, de cada epicôndilo do úmero em direção à extremidade distal do 3 o dedo da mão. A partir da construção desses eixos foram realizadas medidas diretas longitudinalmente aos eixos e medidas perpendicularmente a eles no local. 0 ponto do FUC foi medido a $16,3 \%$ do comprimento do ELM e a $11,4 \%$ da circunferência de sua localização no eixo. 0 ponto do ERC foi medido a $16,3 \%$ do comprimento do ELL e a $17,1 \%$ da circunferência de sua localização no eixo (34).

Solicitou-se que a velocidade de alcance utilizada fosse aquela confortável para o sujeito (17). Após um sinal inicial de "prepara" seguido de "vai", os sujeitos realizavam o alcance e paravam ao atingirem o alvo. Foram realizadas duas práticas e três movimentos gravados, com intervalo de 5 minutos de descanso entre cada um.
A sincronização das filmagens das duas câmeras foi realizada com um flash de câmera fotográfica e a sincronização destas com a eletromiografia foi feita por meio de um cronômetro, logo após o disparo do flash.

\section{Análises}

A coleta dos movimentos de alcance foi realizada conforme os procedimentos descritos anteriormente, com eletromiografia e cinemática simultaneamente. 0 sistema de videogrametria utilizado para a aquisição e o processamento das imagens foi o Digital Video for Biomechanics (Dvideow), desenvolvido no Laboratório de Instrumentação para Biomecânica da Faculdade de Educação Física da Unicamp $(35,36)$. Foi realizada uma análise qualitativa individual de cada alcance e selecionado o melhor movimento para a apresentação da análise quantitativa.

Para a análise eletromiográfica foi utilizado o software WinDaq (DATAQ Instruments) para aquisição dos dados digitalizados por placa de conversão A/D de 16 bits de resolução e frequência de amostragem de $1 \mathrm{KHz}$ em cada canal. 0 tempo de análise da eletromiografia foi o período equivalente ao tempo do movimento observado na cinemática.

Na cinemática foi avaliado o deslocamento em cada coordenada ( $\mathrm{x}$, y e z) de cada articulação (ombro, cotovelo e punho) para o lado não acometido e o acometido de cada sujeito e obtida a média e o desvio-padrão. Na eletromiografia foi avaliado o root mean square (RMS) e o valor máximo, que foi obtido por meio do teste de força máxima manual de cada músculo separadamente. Por meio desses valores, foi calculado o RMS normalizado (RMS/valor máximo) de cada músculo, para o lado não acometido e o acometido de cada sujeito, como pode ser visto na equação a seguir representada:

$$
\mathrm{EMG}_{\mathrm{n}}[\mathrm{UA}]=\frac{\mathrm{RMS}[\mathrm{UA}]}{\text { Máximo CIVM }[\mu \mathrm{V}]}
$$

Os dados foram processados por meio dos softwares Excel e KaleidaGraph, e posteriormente foi utilizado, para a análise estatística, o software Statistica. A estatística descritiva foi utilizada para o cálculo de médias e desvios-padrão. Para os dados cinemáticos foi utilizada a ANOVA, considerando o deslocamento 
como variável dependente e as demais variáveis (sujeitos, lados, articulações e coordenadas) como independentes. Um tratamento similar foi realizado com os dados da eletromiografia, sendo a RMS obtida a variável dependente e as demais variáveis (sujeitos, lados e músculos) selecionadas como independentes. A seguir foram conduzidos também testes post-hoc de Fisher - LSD, separadamente, para as variáveis. 0 nível de significância utilizado foi de $95 \%$.

\section{Resultados}

\section{Apresentação dos casos}

O Sujeito 1, 46 anos, sexo feminino, AVE isquêmico em cápsula interna direita há 6 meses, obteve pontuação na escala Fugl-Meyer de 123/126 para controle dos membros superiores e 100/100 para membros inferiores, totalizando $223 / 226$ pontos. Na escala de deficiência de tronco sua pontuação foi de 16/23, e na escala de Ashworth obteve pontuação 1. Sua dominância era destra enquanto o lado acometido pelo AVE foi o esquerdo. Foram escolhidos o movimento 3 para o lado não acometido e o movimento 2 para o lado acometido, sendo realizados em 1,12 e 1,97 segundos, respectivamente.

Já o Sujeito 2, 56 anos, sexo feminino, AVE hemorrágico do lado direito, porém, não tendo informado o local exato no prontuário há 10 meses, obteve pontuação na escala Fugl-Meyer de $120 / 126$ para controle dos membros superiores e de 95/100 para membros inferiores, totalizando 215/226 pontos. Na escala de deficiência de tronco sua pontuação foi de 20/23 e na escala de Ashworth obteve pontuação 1. Sua dominância era sinistra e o lado acometido pelo AVE foi o esquerdo. Foram escolhidos o movimento 2 para o lado não acometido e o movimento 2 para o lado acometido, sendo realizados em 1,60 e 1,76 segundos, respectivamente.

O Sujeito 3, 47 anos, sexo feminino, AVE isquêmico em cápsula interna esquerda há 6 meses, obteve pontuação na escala Fugl-Meyer de 120/126 para controle dos membros superiores e de 96/100 para membros inferiores, totalizando $216 / 226$ pontos. Na escala de Deficiência de Tronco sua pontuação foi de 23/23 e na escala de Ashworth obteve pontuação 1 . Sua dominância era destra e o lado acometido pelo AVE foi o direito. Foram escolhidos o movimento 3 para o lado não acometido e o movimento 2 para o lado acometido, sendo realizados em 0,72 e 1,06 segundos, respectivamente.

Por fim, o Sujeito 4, 69 anos, sexo masculino, AVE isquêmico frontal lacunar direito há 6 meses, obteve pontuação na escala Fugl-Meyer de $123 / 126$ para controle dos membros superiores e de 88/100 para membros inferiores, totalizando 211/226 pontos. Na escala de Deficiência de Tronco sua pontuação foi de $16 / 23$ e na escala de Ashworth obteve pontuação 0. Sua dominância era destra enquanto o lado acometido pelo AVE foi o esquerdo. Foram escolhidos o movimento 2 para o lado não acometido e o movimento 2 para o lado acometido, sendo realizados em 0,98 e 1,44 segundos, respectivamente.

Dessa forma, pode-se observar que a amostra é homogênea com relação à maioria dos dados avaliados.

\section{Análise cinemática}

Para os dados de deslocamento, houve diferença significativa entre os sujeitos $\left(\mathrm{F}_{(3,63)}=3.081, \mathrm{p}=\right.$ 0.03), mostrando maior variabilidade no Sujeito 3 por meio da análise post-hoc (Sujeito $1 \times$ Sujeito 3 $\mathrm{p}=0.01$ e do Sujeito $3 \times$ Sujeito $4 \mathrm{p}=0.02$ ). Não houve diferença significativa entre os lados $\left(\mathrm{F}_{(1,63)}=\right.$ $1.441, \mathrm{p}=0.23$ ), mesmo com deslocamento um pouco maior do lado não acometido em comparação com o lado acometido. Com relação às articulações, o deslocamento de cada uma teve diferença significativa $\left(\mathrm{F}_{(2,63)}=27.496, \mathrm{p}=0.00\right)$, mostrando, ainda, menor deslocamento na articulação do ombro na análise post-hoc $(\mathrm{p}=0.00)$. Houve diferença significativa entre as coordenadas $\mathrm{x}$, y e $\mathrm{z}\left(\mathrm{F}_{(2,63)}=36.702\right.$, $\mathrm{p}=0.00$ ) tendo a última menor deslocamento mostrado na análise post-hoc $(\mathrm{p}=0.00)$.

0 Gráfico 1 mostra a média e o desvio-padrão entre todos os indivíduos, com relação ao deslocamento das articulações do ombro, cotovelo e punho, lado não acometido e acometido. Observa-se maior deslocamento das articulações do cotovelo e punho nas coordenadas $\mathrm{x}$ e $\mathrm{y}$.

Houve diferença no tempo de execução dos movimentos quando comparado o lado não acometido com o acometido (Tabela 2), evidenciando a lentificação desse último. 

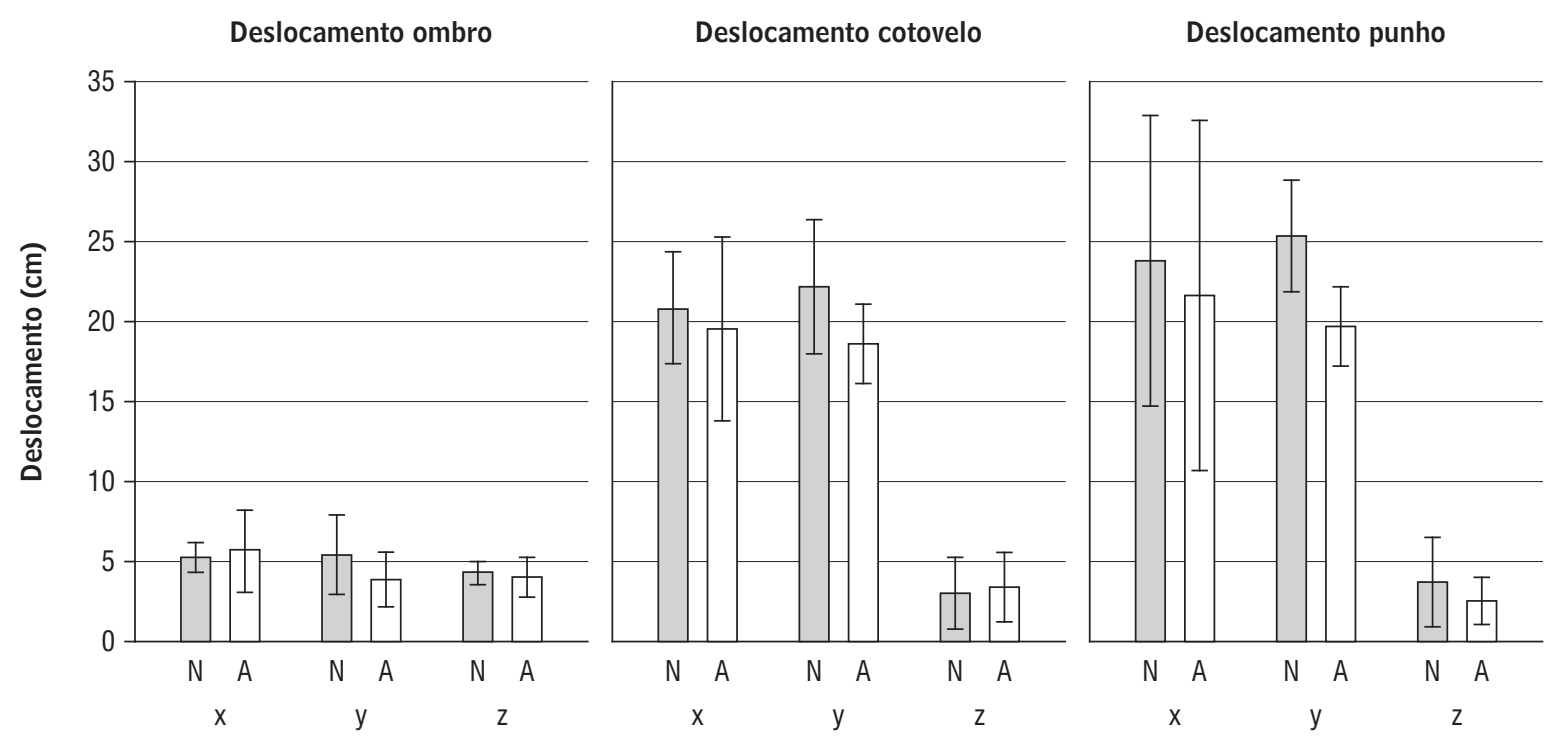

Gráfico 1 - Deslocamento da articulação do ombro, do cotovelo e do punho nas coordenadas x, y e z dos lados não acometido e acometido

Legenda: $\mathrm{N}$ = lado não acometido; $\mathrm{A}=$ lado acometido.

Tabela 2 - Tempo total de um alcance determinado pela chegada ao alvo

\begin{tabular}{lcc}
\hline & \multicolumn{2}{c}{ Tempo (s) } \\
\cline { 2 - 3 } Sujeito & $\begin{array}{c}\text { Lado } \\
\text { normal }\end{array}$ & $\begin{array}{c}\text { Lado } \\
\text { acometido }\end{array}$ \\
\hline 1 & 1,12 & 1,97 \\
2 & 1,6 & 1,76 \\
3 & 0,72 & 1,06 \\
4 & 0,98 & 1,44 \\
Média \pm desvio-padrão & $1,1 \pm 0,3$ & $1,6 \pm 0,3$ \\
\hline
\end{tabular}

\section{Análise eletromiográfica}

$\mathrm{Na}$ análise dos dados eletromiográficos, não houve diferença significativa entre os sujeitos $\left(\mathrm{F}_{(3,31)}=\right.$ 2.437, $\mathrm{p}=0.08)$, entre os lados $\left(\mathrm{F}_{(1,31)}=3.384, \mathrm{p}=\right.$ $0.07)$ e entre os músculos $\left(\mathrm{F}_{(4,31)}=0.942, \mathrm{p}=\right.$ $0.45)$. Nesse último, observaram-se valores mais altos e maior variabilidade na atividade muscular do trapézio.

O Gráfico 2 mostra a média e o desvio-padrão de todos os sujeitos do RMS normalizado de cada músculo nos lados não acometido e acometido.

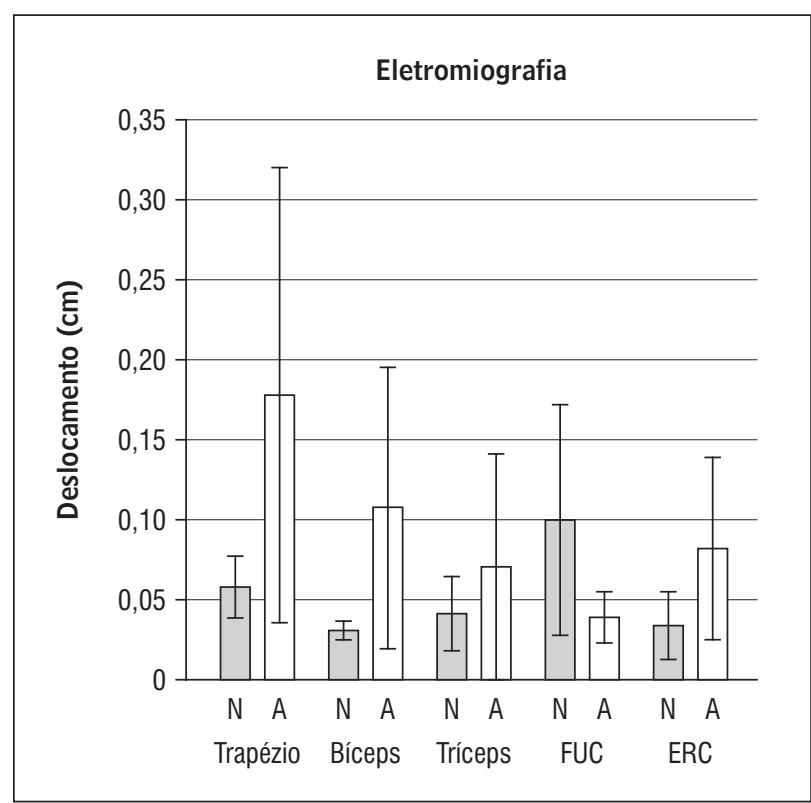

Gráfico 2 - Média e desvio-padrão do RMS normalizado para cada músculo dos lados não acometido e acometido Legenda: $\mathrm{N}$ = lado não acometido; $\mathrm{A}$ = lado acometido.

\section{Discussão}

O objetivo deste estudo foi realizar uma análise cinemática e eletromiográfica do movimento de alcance funcional em indivíduos hemiparéticos 
com diagnóstico de acidente vascular encefálico. 0 movimento de alcance foi utilizado por ser um componente fundamental de muitas atividades da vida diária; por exigir a movimentação coordenada de múltiplos segmentos da extremidade superior; e para entender melhor o controle motor de pessoas com hemiparesia crônica durante esta tarefa (14). Este estudo envolveu as características específicas do alcance, pois uma melhor compreensão das características do movimento funcional fornecerá informações adicionais que poderão ajudar a orientar a escolha de técnicas de reabilitação e parâmetros de exercícios.

Dentre os estudos envolvendo a cinemática do movimento de alcance em pacientes com hemiparesia pós-AVE, destaca-se o estudo de Wu et al (24), que investigaram o movimento do membro superior após terapia de restrição. Essa pesquisa mostrou que a terapia de restrição comparada à terapia convencional melhorou o desempenho cinemático. Uma limitação dessa pesquisa foi a ausência de dados eletromiográficos para fornecer uma imagem mais completa dos parâmetros do movimento. Já o presente estudo fornece tanto dados cinemáticos como eletromiográficos para avaliação do movimento de alcance.

Cacho, de Melo e de Oliveira (31) avaliaram e estabeleceram o acompanhamento motor de pacientes hemiplégicos por meio do protocolo de desempenho físico de Fugl-Meyer. Nesse trabalho foi observado que a amplitude articular do movimento do ombro é dificultada pela dor; o déficit somatossensorial prejudica o controle motor do membro afetado; e o sinergismo anormal muitas vezes é forte o suficiente para impedir os movimentos isolados fora dos padrões sinergísticos em massa. No recente estudo, seguindo a mesma linha, foi utilizada essa escala, estabelecendo pontuação menor que 50 (pacientes com nível severo) como critério de exclusão. Portanto, os participantes desta pesquisa demonstraram nível baixo de comprometimento e não apresentaram tão evidentemente as características citadas pelos autores, as quais poderiam influenciar na realização do movimento.

Há divergências na literatura sobre a contenção ou não do tronco durante a reabilitação do membro superior. Uma vez que indivíduos saudáveis alcançam um objeto colocado na distância de alcance do braço, ocorre uma boa coordenação entre os movimentos do cotovelo e do ombro, enquanto o tronco é praticamente imóvel. Michaelsen et al.(18), em estudos anteriores em pacientes hemiparéticos, descrevem excessivo movimento do tronco e da cintura escapular em movimentos de alcance para preensão em alvos colocados perto do corpo. Recentemente, foi sugerido, no entanto, que esse comportamento pode limitar o potencial de recuperação do movimento normal do braço. Reduzir mecanismos compensatórios, limitando o deslocamento do tronco, pode encorajar o regresso dos padrões típicos observados em indivíduos saudáveis (32). No presente estudo, foi utilizada a contenção de tronco para determinar mais claramente cada componente do movimento do membro superior, eliminando movimentos compensatórios.

Thielman (32) verificou maior dificuldade de realização do alcance em indivíduos hemiparéticos no alvo localizado ipsilateral ao lado afetado. Não foi utilizada essa localização do alvo, pois, segundo Thielman, a realização de testes com esses alvos gera fadiga e diminui o desempenho durante a realização do movimento. Dessa forma, os indivíduos conseguiram realizar todos os alcances eficientemente, sem gerar fadiga, já que o alvo foi localizado funcionalmente na linha do ombro a ser avaliado.

Conforme o esperado para a tarefa proposta, o deslocamento do ombro foi menor que o das outras articulações. 0 ombro realiza a função de estabilização do membro superior durante a tarefa do alcance enquanto o cotovelo aproxima e afasta a extremidade distal do membro superior para a realização de alcance e preensão de objeto. Isso ocorre como descrito por Shumway-Cook (37), em que o alcance envolve a ativação adequada dos músculos, a fim de estabilizar a escápula, a caixa torácica e a cabeça umeral durante esse movimento, e essa ativação dos músculos das articulações do ombro, do cotovelo e do punho permite o transporte do braço.

Na coordenada $\mathrm{z}$, o deslocamento se diferenciou das demais, pois nessa tarefa não houve necessidade de adução ou abdução horizontal do ombro destacada nessa coordenada. Além disso, os sujeitos obtiveram pontuação menor ou igual a 1 na escala de Ashworth, o que não demonstra hipertonia adutora, que provocaria o deslocamento na coordenada $\mathrm{z}$. E, ainda, nenhum sujeito apresentou dismetria ou tremor, que também favoreceria deslocamentos em z. De acordo com Shumway-Cook (37), o movimento de alcance e preensão normal exige: rotação escapular, movimento adequado da cabeça umeral, capacidade 
de supinação do braço, flexão de ombro e cotovelo, capacidade de estender o punho ligeiramente além da posição neutra e mobilidade suficiente da mão para permitir a preensão e o desprendimento.

A diferença de deslocamento encontrada no Sujeito 3 não se relacionou com nenhum dado avaliado (dominância, idade, tempo pós-AVE, tipo de AVE, local de AVE, pontuação Fugl-Meyer, EDT e Ashworth), inclusive com a eletromiografia. 0 único dado diferente dos outros sujeitos foi o menor tempo de realização do movimento para o Sujeito 3. Van Vliet (17) investigou a coordenação dos componentes do alcance funcional em indivíduos hemiparéticos e saudáveis, utilizando velocidades de alcance preferencial e o mais rápido possível. Dessa forma, ele verificou que, quando a variabilidade temporal diminui com movimentos mais rápidos, a variabilidade espacial pode aumentar porque há menos tempo para fazer as correções baseadas no feedback visual.

A variabilidade de deslocamento foi maior do lado parético, o que pode ser interpretado como uma inconsistência no controle motor. Stoeckmann et al. (38) realizaram uma avaliação eletromiográfica do membro superior de dez hemiparéticos pósAVE e dez sujeitos saudáveis no exercício resistido, utilizando Thera-Band ${ }^{\circledR}$, e obtiveram como resultado que a ativação muscular foi significativamente maior em praticamente todas as medidas nos membros superiores paréticos e não paréticos dos participantes com AVE do que nos participantes do controle, que são saudáveis. Yarosh et al. (39) apresentaram dados semelhantes após avaliarem os movimentos e a atividade elétrica do punho em sete indivíduos com AVE e sete saudáveis, em que encontraram comprometimento tanto do lado parético como do lado não parético em comparação com saudáveis. Nossos resultados corroboram os de Yarosh et al. e os de Stoeckmann et al., sugerindo que os sujeitos hemiparéticos têm padrões anormais de ativação muscular, inclusive no membro não parético, o que pode explicar a diferença não significativa de nossos resultados.

Em relação à quantidade de ativação muscular avaliada, não houve diferença significativa. Entretanto, a ativação muscular do trapézio foi maior no lado acometido que no não acometido e este deveria ser ativado apenas como estabilizador do movimento. Para elucidar o exposto, pode-se fazer referência ao posicionamento de Gowland (40), que considera que há um recrutamento inadequado de unidades motoras nessa população. Gowland avaliou a eletromiografia de 44 pacientes com hemiplegia após acidente vascular cerebral e dez indivíduos sem comprometimento para examinar as contribuições da unidade de recrutamento motor inadequado no movimento do braço hemiplégico e concluiu que este pode ser a causa das desordens no membro superior desses indivíduos.

O comportamento diferenciado do FUC, em que a ativação do lado acometido foi menor que no lado não acometido, pode ser explicado pelo posicionamento dos eletrodos. Como não há determinações do Protocolo de Seniam para esse músculo, foi utilizado o estudo de Lima et al. (34). Como a região anterior do antebraço é uma área de origem dos flexores de punho e dedos, o posicionamento do eletrodo para esse estudo possivelmente não isolou a atividade do músculo solicitado. Assim, o máximo conseguido no teste de força máxima foi maior do lado acometido e, portanto, ao se obter o RMS normalizado, por ser inversamente proporcional, esse valor foi reduzido.

As análises cinemática e eletromiográfica, de um modo geral, mostraram resultados semelhantes aos encontrados na literatura. No entanto, este estudo acrescenta uma visão mais sistematizada do movimento de alcance, considerando também seus aspectos funcionais, diferenciando dos demais estudos de alcance em hemiparéticos, por apresentar uma avaliação concomitante de cinemática e eletromiografia. São necessários estudos com maior quantidade de sujeitos para melhorar a sensibilidade às diferenças estatísticas dos testes utilizados, repetir a análise após intervenções específicas, comparar sujeitos hemiparéticos com sujeitos saudáveis, além de avaliar sujeitos com hemiparesia crônica.

\section{Conclusão}

O movimento de alcance realizado com o membro superior parético e o não parético de pacientes pós-acidente vascular encefálico demonstra diferenças relacionadas ao tempo de realização da tarefa, sendo o parético maior que o não parético, e à amplitude do movimento, que foi maior do lado não parético. A pequena amostra não permite a generalização dos achados, mas apresenta, certamente, uma contribuição na área de pesquisa na reabilitação da função de membro superior no hemiparético. 


\section{Referências}

1. American Heart Association. Heart disease and stroke statistics update; 2005.

2. Mayo NE, Wood-Dauphinee S, Ahmed S, Gordon C, Higgins J, McEwen S, et al. Disablement following stroke. Disabil Rehabil. 1999;21(5-6):258-68.

3. Hunter S, Crome P. Hand function and stroke. Rev Clin Gerontol. 2002;12(1):68-81.

4. Blennerhassett JM, Carey LM, Matyas TA. Grip force regulation during pinch grip lifts under somatosensory guidance: comparison between people with stroke and healthy controls. Arch Phys Med Rehabil. 2006;87(3):418-29.

5. Hermsdorfer J, Marquardt C, Wack S, Mai N. Comparative analysis of diadochokinetic movements. J Electromyogr Kinesiol. 1999;9(4):283-95.

6. Lang CE, Wagner JM, Bastian AJ, Edwards DF, Sahrmann SA. Deficits in grasp versus reach during acute hemiparesis. Exp Brain Res. 2005;166(1):126-36.

7. Mai N. Residual control of isometric finger forces in hemiparetic patients. Evidence for dissociation of performance deficits. Neurosci Lett. 1989;101(3):347-51.

8. McDonnell MN, Hillier SL, Ridding MC, Miles TS. Impairments in precision grip correlate with functional measures in adult hemiplegia. Clin Neurophysiol. 2006;117(7):1474-80.

9. Nowak DA. Different modes of grip force control: voluntary and externally guided arm movements with a hand-held object. Clin Neurophysiol. 2004;115(4): 839-48.

10. Nowak DA, Grefkes C, Dafotakis M, Küst J, Karbe H, Fink GR. Dexterity is impaired at both hands following unilateral subcortical middle cerebral artery stroke. Eur J Neurosci. 2007;25(10):3173-84.

11. Roby-Brami A, Jacobs S, Bennis N, Levin MF. Hand orientation for grasping and arm joint rotation patterns in healthy subjects and hemiparetic stroke patients. Brain Res. 2003;969(1-2):217-29.

12. Jeannerod M. The timing of natural prehension movements. 1984;16(3):235-54.

13. Jeannerod M. The neural and behavioural organization of goal-directed movements. Oxford: Oxford University Press; 1988.
14. Wagner JM, Lang CE, Sahrmann SA, Edwards DF, Dromerick AW. Sensorimotor impairments and reaching performance in subjects with post stroke hemiparesis during the first few months of recovery. Phys Ther. 2007;87(6):751-65.

15. Cirstea MC, Levin MF. Compensatory strategies for reaching in stroke. Brain. 2000;123(Pt 5):940-53.

16. Kamper DG, McKenna-Cole AN, Kahn LE, Reinkensmeyer DJ. Alterations in reaching after stroke and their relation to movement direction and impairment severity. Arch Phys Med Rehabil. 2002;83(5):702-7.

17. van Vliet PM, Sheridan MR. Coordination between reaching and grasping in patients with hemiparesis and healthy subjects. Arch Phys Med Rehabil. 2007; 88(10):1325-31.

18. Michaelsen SM, Jacobs S, Roby-Brami A, Levin MF. Compensation for distal impairments of grasping in adults with hemiparesis. Exp Brain Res. 2004;157(2): 162-73.

19. Gaimmi M, Carda S, Giovanazana C. Using kinematic analysis to evacuate constraint-induced movement therapy in chronic stroke patients. Neurorehabil Neural Repair. 2008;22(1):31-9.

20. Kahn LE, Zygman ML, Rymer WZ, Reinkensmeyer DJ. Robot-assisted reaching exercise promotes arm movements recovery in chronic hemiparetic stroke: a randomized controlled pilot study. J Neuroeng Rehabil. 2006;3:12.

21. Michaelsen SM, Levin MF. Short-term effects of practice with trunk restraint on reaching movements in patients with chronic stroke: a controlled trial. Stroke. 2004;35(8):1914-9.

22. Summers JJ, Kagerer FA, Garry MI. Bilateral and unilateral movement training on upper limb function in chronic stroke patients: a TMS study. J Neurol Sci. 2007;252(1):76-82.

23. Thielman GT, Dean CM, Gentile AM. Rehabilitation of reaching after stroke: task-related training versus progressive resistive exercise. Arch Phys Med Rehabil. 2004;85(10):1613-8.

24. Wu CY, Chen CL, Tang SF, Lin KC, Huang YY. Kinematic and clinical analyses of upper-extremity movements after constraint-induced movement therapy in patients with stroke: a randomized controlled trial. Arch Phys Med Rehabil. 2007;88(8):964-70. 
25. Bevilaqua-Grossi D, Felicio LR, Simões R, Coqueiro KRR, Monteiro-Pedro V. Avaliação eletromiográfica dos músculos estabilizadores da patela durante exercício isométrico de agachamento em indivíduos com síndrome da dor femoropatelar. Rev Bras Med Esporte. 2005;11(3):159-63.

26. Ervilha UF, Duarte MA, Amadio AC. Cinemática da articulação do joelho e atividade eletromiográfica de músculos do membro inferior durante a marcha em ambiente aquático e terrestre. Rev Bras Biomec. 2002;3(4):21-6.

27. Silva SRD, Gonçalves M. Comparação de protocolos para avaliação da fadiga muscular pela eletromiografia de superfície. Motriz. 2003;9(1):51-8.

28. Oliveira AS, Freitas CMS, Monaretti FH, Ferreira F, Noguti R, Bérzin F. Avaliação eletromiográfica de músculos da cintura escapular e braço durante a realização de exercícios com extremidade fixa e carga axial. Rev Bras Med Esporte. 2008;14(5):466-71.

29. Hughes AM, Freeman CT, Burridge JH, Chappell PH, Lewin PL, Rogers E. Shoulder and elbow muscle activity during fully supported trajectory tracking in people who have had a stroke. J Electromyogr Kinesiol. 2010;20(3):465-76.

30. Barker RN, Brauer S, Carson R. Training-induced changes in the pattern of triceps to biceps activation during reaching tasks after chronic and severe stroke. Exp Brain Res. 2009;196(4):483-96.

31. Cacho EWA, Melo FRLV, Oliveira R. Avaliação da recuperação motora de pacientes hemiplégicos através do protocolo de desempenho físico Fugl-Meyer. Revista Neurociências. [online] 2004 [acesso em 26 nov. 2010];12(2). Disponível em: http://www.revista neurociencias.com.br/edicoes/2004/RN\%2012\%20 02/Pages\%20from\%20RN\%2012\%2002-8.pdf

32. Thielman G, Kaminski T, Gentile AM. Rehabilitation of reaching after stroke: comparing 2 training protocols utilizing trunk restraint. Neurorehabil Neural Repair. 2008;22(6):697-705.

33. Hermes HJ, Freriks B, Disselhorst-Klug C, Rau G. Development of recommendation for SMG sensors and sensors placement procedures. J Electromyography Kinesiology. 2000;10:361-74.
34. Lima FM, Abreu RS, Shimano GN, Souza LAPS, Fernandes LFRM. Methodological proposal for surface electrodes positioning on the wrist and fingers muscles through anthropometric measurements of forearm. Brazilian J Oral Sciences (Online). 2010;9(4): 208-8.

35. Barros RML, Brenkizikofer R, Leite NJ, Figueroa PJ. Desenvolvimento e avaliação de um sistema para análise cinemática tridimensional de movimentos humanos. Rev Bras Eng Bioméd. 1999;15(1-2):79-86.

36. Figueroa PJ, Leite NJ, Barros RML. A flexible software for tracking of markers used in human motion analysis. Computer Methods and Programs in Biomed. 2003;72(2):155-65.

37. Shumway-Cook A, Woollacott MH. Controle motor: teoria e aplicação prática. 2a ed. São Paulo: Manole; 2002.

38. Stoeckmann TM, Sullivan KJ, Scheidt RA. Elastic, viscous and mass load effects on poststoke muscle recruitment and co-contraction during reaching: a pilot study. Phys Ther. 2009;89(7):665-78.

39. Yarosh CA, Hoffman DS, Strick PL. Deficits in movements of the wrist ipsilateral to a stroke in hemiparetic subjects. J Neurophysiol. 2004;92(6):3276-85.

40. Gowland C, Debruin H, Basmanjian JV, Plews N, Brucea I. Agonist and antagonist activity during voluntary upper-limb movement in patients with stroke. Phys Ther. 1992;72(9):624-33.

Recebido: 01/12/2010

Received: 12/01/2010

Aprovado: $20 / 12 / 2010$

Approved: $12 / 20 / 2010$ 